\title{
Pore Type and Porosity Distribution of Carbonate Reservoir Based on 3D Seismic Inversion in "P" Field Salawati Basin
}

\author{
Avishena Prananda ${ }^{1}$, Mohammad Syamsu Rosid ${ }^{1, *}$, and Robet Wahyu Widodo ${ }^{2}$ \\ ${ }^{1}$ Department of Physics, Faculty of Mathematics and Natural Sciences, University of Indonesia, Depok - Indonesia \\ ${ }^{2}$ PT. Pertamina Upstream Technical Center, Kwarnas Pramuka, Jakarta-Indonesia
}

\begin{abstract}
Overall, carbonate rock has complex and more heterogeneous physical characteristic, compared to siliciclastic sedimentary rock. One parameter, which distinguishes carbonate rock and siliciclastic is pore structure/pore type. The heterogeneity and complexity of carbonate reservoir pore type are affected by the sedimentation process and the diagenesis process. Pore type classification is divided into three: interparticle, stiff, and crack. Therefore, carbonate pore type determination becomes important to enhance drilling success. This paper explains pore types prediction, porosity, and acoustic impedance on carbonate reservoir. The Differential Effective Medium (DEM) method to analyze carbonate reservoir pore type has been applied. DEM method generates bulk and shear modulus parameters to create carbonate $V p$ and $V s$ model based on pore type. We also do a 3D seismic inversion to create acoustic impedance distribution, porosity, and pore type. Afterward, we made cube porosity and pore type cube by using geostatistics method to provide a better result. Moreover, this study shows low impedance value correlates with high porosity value and enhancement of porosity value correlates with crack and interparticle pore type on "P" field, Salawati Basin.
\end{abstract}

Keywords: Differential Effective Medium; Pore Type; Carbonate; Salawati Basin.

\section{Introduction}

Kais Carbonate reef is a carbonate reservoir that producing a reservoir in the Salawati Basin. Since 1936, Salawati Basin has produced more than $400 \mathrm{MMBO}$ and 200 BCFG through 2014 [1]. But it is successful in small scale and difficult exploration, so a new exploration concept is needed to characterize carbonate reservoirs [2]

Identifying the pore type of carbonate reservoir is a new exploration concept. The pore type of carbonate reservoir is more complex and heterogeneous than the siliciclastic rock pore type, which is as a result of locally deposited environments and diagenetic processes such as cementation and dissolution. In siliciclastic pore type, it tends to be homogeneous, whereas in carbonate reservoirs the pore type is divided into three types namely stiff, interparticle, and crack [3]. The complexity and heterogeneity of reservoir carbonate pore types have implications if the velocity of seismic waves propagates in carbonate rock reservoirs. In 1999, a study to predict pore type in carbonate showed pore type controls porosity from acoustic velocity deviations [4].

The secondary pore type reservoir carbonate can be determined by various methods, one of them is the Differential Effective Medium (DEM) method, where geometry aspects are determined based on Zhao's classification.

In 2016 a comparative study was conducted on the method of determining the pore type Differential Effective Medium (DEM) with Kuster-Toksoz (KT).
The DEM method was considered better than the KT method to estimate the pore type of carbonate reservoir [5]. Therefore, we applied the DEM method to characterize carbonate reservoirs. The principle of DEM method is input porosity fraction and fraction mineral content and the output is rock modulus as the function porosity to create $V p$ and $V S$ model [6].

The $V p$ model will compare with the $V p$ measurement to get pore type in one dimension (1D). For analyzing pore type lateral and vertical distribution is in threedimension (3D). 3D post stack seismic inversion method was applied as a trend input for geostatistical method.

This study used 3 well logs and post-stack 3D seismic data, as well as a thin section from a carbonate reservoir in the "P" field, which is located in the Salawati Basin. Salawati Basin has been controlled by Sorong Fault since Early Pliosen [7]. Stratigraphically, sequences in Salawati were formed since Paleozoikum to Kenozoikum. Tertiary sediments have been deposited since Late Eocene to Early Pliocene. The study, specifically observed carbonate Kais in the Miocene time. During this time, sea level receded to the west, because the eastern basin was being uplifted [8]. All those data are used to analyze Kais carbonate (Fig. 1) pore type distribution.

* Corresponding author: syamsu.rosid@ui.ac.id 


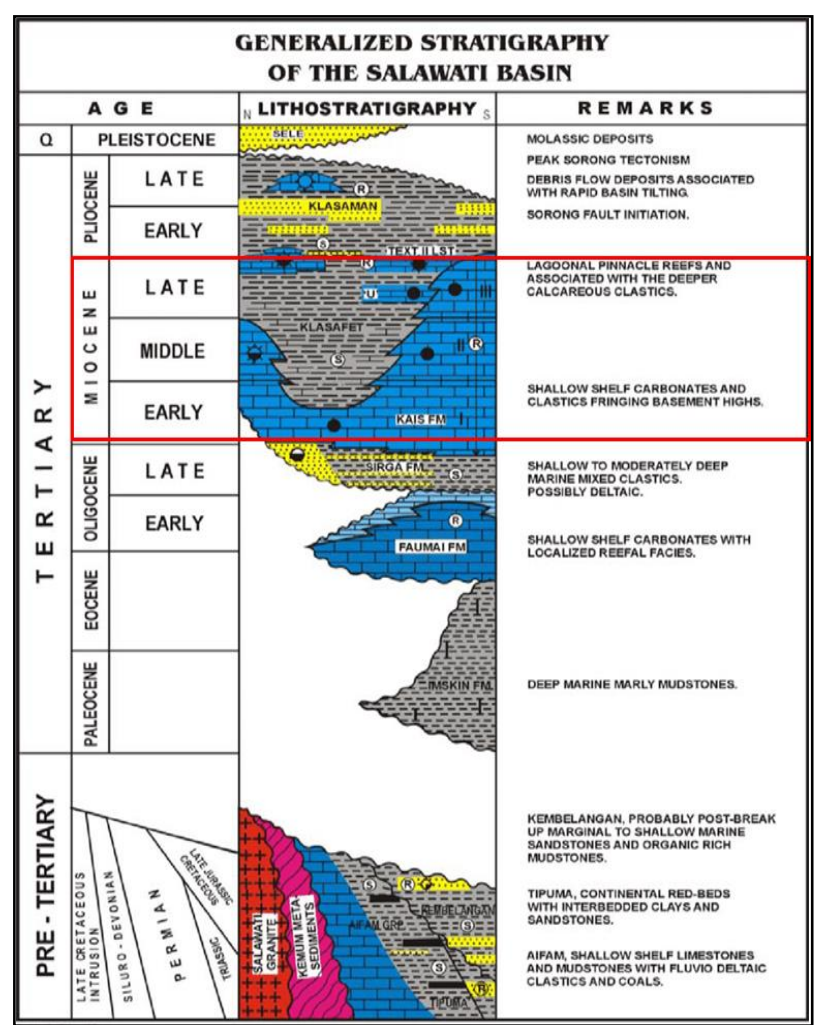

Fig. 1. Stratigraphy of the Salawati Basin [8].

\section{Method}

Elastic properties modelling successfully applied in siliciclastic rocks, Xu-Payne extended the $\mathrm{Xu}$-White model, which the total pore space of carbonate is classified into four types i.e: clay-related pores, interparticle pores, microcrack, and stiff pores [9]. Step to get the pore type distribution through several stages such as Rock Physic, Post-Stack Seismic Inversion, and Petrophysical Modelling Method.

\subsection{Rock Physic Method}

The first step rock physics method is to model rock modulus by calculating the mineral fraction and mineral modulus using the Voigt-Reuss-Hill method, note that the solid rock does not have pores.

$$
\begin{gathered}
M v=\sum_{i-1}^{n} M i . f i \\
\frac{1}{M r}=\sum_{i-1}^{n} \frac{f i}{M i} \\
M v r h=\frac{M v+M r}{2}
\end{gathered}
$$

where:

$M r$ : Elastic modulus of Reuss method

$M v$ : Elastic modulus of Voigt method

$M_{\mathrm{i}}: \mathrm{i}^{\text {th }}$ elastic modulus mineral

$f_{\mathrm{i}}: \mathrm{i}^{\text {th }}$ mineral fraction
In this step, equation 3 is average equation 1 and equation 2. In the next step, we applied Wood's Relation to get the elastic modulus value of fluid within rock by using equation 4.

$$
\frac{1}{K f}=\frac{\text { Swater }}{\text { Kwater }}+\frac{\text { Soil }}{\text { Koil }}+\frac{\text { Sgas }}{\text { Kgas }}
$$

The last step of the rock physics method is the DEM method. It models the bulk and shear modulus saturated by the inclusion of porosity, fluid, and aspect ratio. The aspect ratio used in the DEM method is Zhao's classification. The aspect ratio of cracks ranges from $0.01-0.02$. The aspect ratio of stiff pores range from 0.7 -0.8 , and the aspect ratio of interparticle pores is range from $0.12-0.15$.

$$
\begin{aligned}
& (1-y) \frac{d}{d y}\left[K^{*}(y)\right]=\left(K_{2}-K^{*}\right) P^{(* 2)}(y) \\
& (1-y) \frac{d}{d y}\left[\mu^{*}(y)\right]=\left(\mu_{2}-\mu^{*}\right) Q^{(* 2)}(y)
\end{aligned}
$$

$K$ : Matrix bulk modulus,

$\mu$ : Matrix shear modulus,

$K_{2}$ : Bulk modulus of inclusion phase,

$\mu_{2}$ : Shear modulus of inclusion phase,

$P^{(* 2)}$ and $\left.Q^{(* 2)}\right)$ : Geometrical aspect from aspect ratio of the initial conditions,

$y$ : Porosity,
$d y$ : Incremental inclusion porosity.

The bulk and shear modulus are used to create $V p$ reference when the pore is full interparticle. The $V p$ interparticle (reference) will be compared to the value of $V p$ measurement. The $V p$ reference is greater than $V p$ measurement indicates crack and interparticle pore, while $V p$ reference less than $V p$ measurement indicates stiff+interparticle pore.

\subsection{Post-Stack Seismic Inversion Method}

The Post-Stack seismic inversion method is a technique to transform stacked data into a quantitative rock physic parameter. The results of inversion methods are acoustic impedance (AI), $V p$, and density. Acoustic impedance is a density product and $V p$, which varies among different rock layers. The $V p$ reference and $V p$ measurement from the rock physic method are used to create acoustic impedance (AI) volume.

\subsection{Petrophysical Modelling Method}

The result of seismic inversion is used to find acoustic impedance deviation. The AI reference greater than AI measurement indicates crack and interparticle pore, while AI reference less than AI measurement is indicating stiff+interparticle pore. The results were normalized to get a percentage ratio to make it easier to understand. The pore type ratio percentage of seismic 
inversion is used as a trend (secondary variable) to distribute pore type with geostatistics. The geostatistical method used is Sequential Gaussian Simulation (SGS). The SGS is an algorithm that simulates nodes in sequence then uses simulations value as conditioning. The basic steps in petrophysical modelling, make a 3D grid-based on depth structure map, reservoir zoning and layering to obtain detailed distribution. Therefore, scale up well $\log$ data becomes a layer evenly distributed with $\log$ data. The next step is resample pore type percentage ratio of seismic inversion, which will be used as the trend (secondary variable) for lateral and vertical distribution of carbonate's pore types. The last step applied petrophysical modeling to distribute pore types by determining the azimuth direction of the input data. The input is well log property, the post stack seismic inversion, and azimuth direction distribution as the trend (secondary variable). Then, the cube volume of carbonate's pore types is obtained.

\section{Result and Discussion}

\subsection{Crossplot of $V p-V s$ Correlation}

Parameter determination in rock physic modeling is based on field data condition (Table 1). The parameter is in bulk and shear modulus form from containing mineral and fluid inside the rock. Result of rock physic modeling ( $V p$ DEM) on $V p$ measurement shows good result. It is proved from high correlation coefficient and low RMSE from three wells. RMS-1 well has correlation coefficient in amount 0.9994, RMSE 0.02042 (Fig.2), RMS-2 well its correlation coefficient is 0.9725 , RMSE 0.07837 (Figure.3), and SAP-1 well, has correlation coefficient in amount of 0.9939 , RMSE 0.05366 . While on correlation crossplot between $V p$ DEM and $V s$ DEM, it has high correlation coefficient and low RMSE. However its relation is not linear, but quadratic polynomial as shown in Figure 5 to Figure 7. RMS-1 well has correlation coefficient 0.9942, RMSE 0.03418 (Figure 5), RMS-2's correlation coefficient is 0.9755 , RMSE 0.06404 (Figure 6), and SAP-1 well's correlation coefficient is 0.9199, RMSE 0.09754. If $V p$ and $V s$ relation is quadratic, therefore reservoir is dominated by Limestone [10].

Table 1. Modification of Elastic Modulus Medium

\begin{tabular}{|c|c|c|}
\hline Mineral and Fluid & $\begin{array}{c}\text { Bulk Modulus } \\
(\mathrm{Gpa})\end{array}$ & $\begin{array}{c}\text { Shear } \\
\text { Modulus } \\
(\mathrm{Gpa})\end{array}$ \\
\hline Clay & 21 & 7 \\
\hline Limestone (Calcite) & 76.8 & 32 \\
\hline Water & 2.314 & 0 \\
\hline Oil & 0.889 & 0 \\
\hline Gas & 0.093 & 0 \\
\hline
\end{tabular}

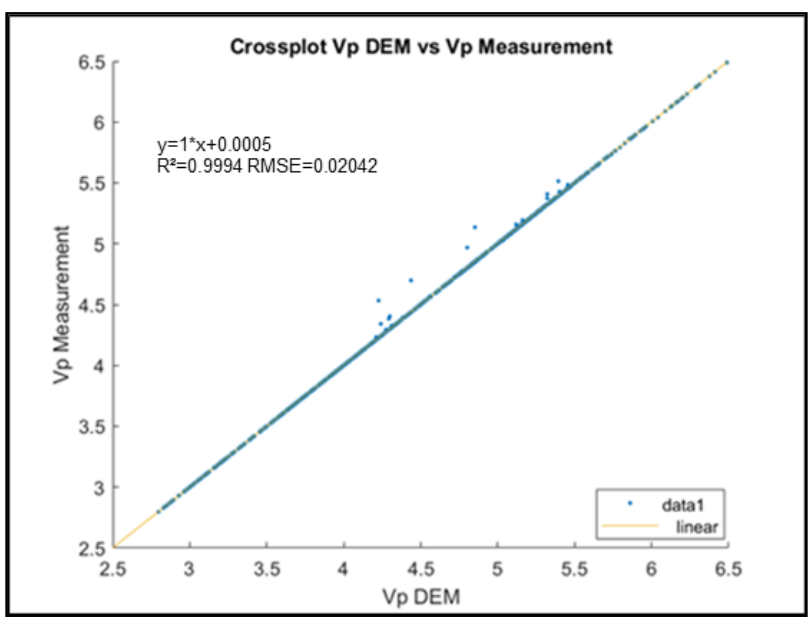

Fig. 2. Cross plot of $V p$ correlation at RMS-1 well

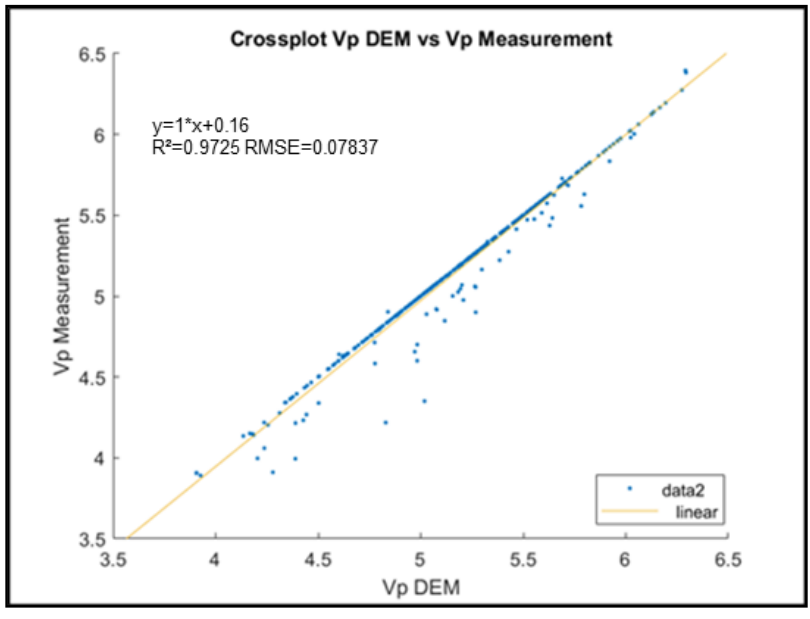

Fig. 3. Crossplot of $V p$ correlation at RMS-2 well

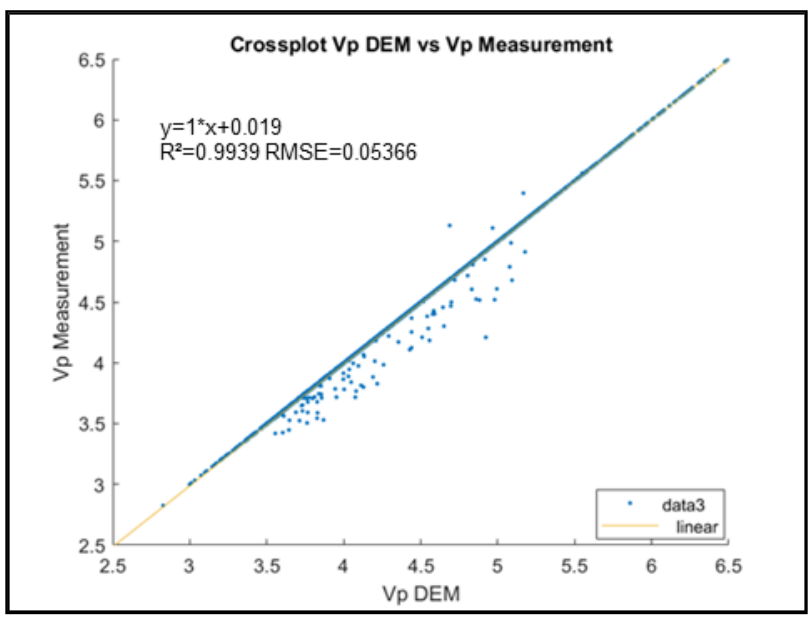

Fig. 4. Cross plot of $V p$ correlation at SAP-1 well

Based on the cross plot results, it can be concluded that input parameter determination in the rock physic model is fit enough to estimate this field. Also, the DEM method can be used to estimate $V s$ value which has not $V s$ data where $V p$ correlation values as control. 


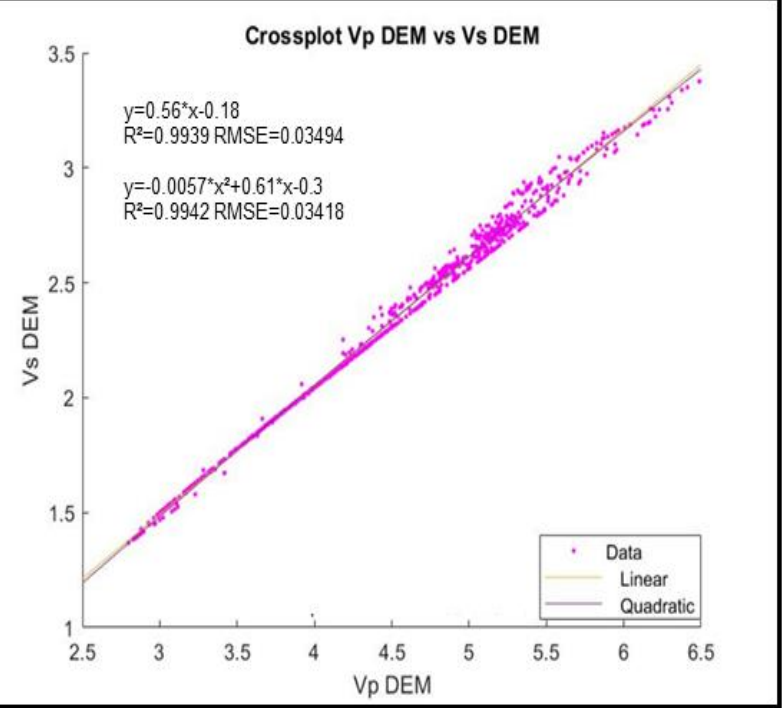

Fig. 5. Cross plot of $V p-V s$ correlation at RMS-1 well

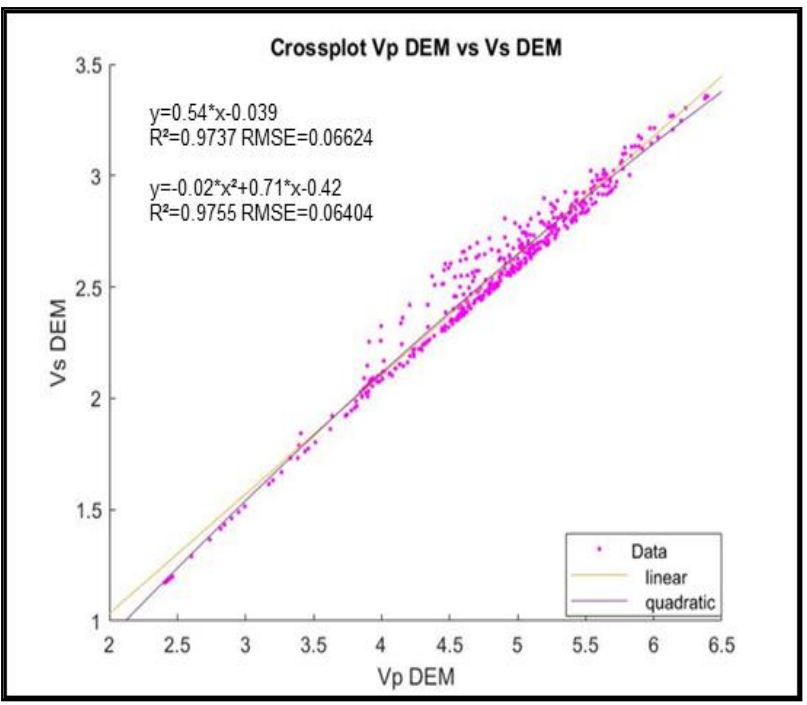

Fig. 6. Cross plot of $V p-V s$ correlation at RMS-2 well

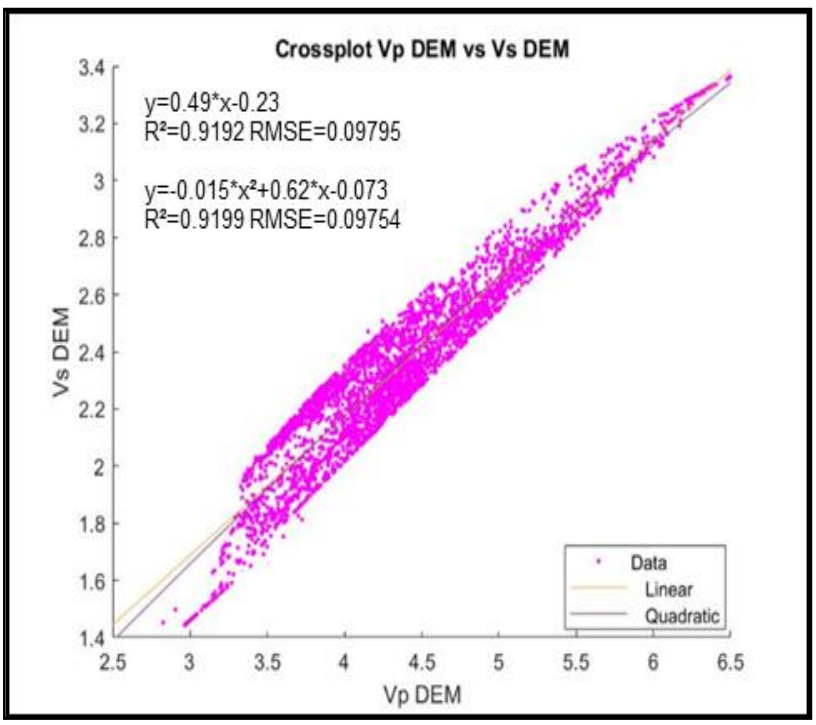

Fig. 7. Cross plot of $V p-V S$ correlation at SAP-1 well

\subsection{Pore Type Quantification}

Fig. 8 and Fig. 9, as well as Figure 10, show the linkage and correlation between clay log volume, porosity log, and Acoustic Impedance log on the Kais Formation pore type log. Further analysis on crack and interparticle (red and white) pore type show significant changes in form of porosity value enhancement and acoustic impedance value is lowered. It is due to crack pore, which can connect pores in a carbonate reservoir. Crack/fracture pore type can be caused by the tectonic process (fold/fault), compaction differences, or exceeding fluid pressure. Moreover, it produces porosity value enhancement in the reservoir. RMS-1 well is producing well with oil and gas containment.

On Fig. 8, the layer is dominated by crack and interparticle pore type, which is affected by tectonic and exceeding fluid containment. RMS-1 well in 4171- 4186 $\mathrm{ft}$ shows increasing the value of porosity significantly and acoustic impedance decreases. It is indicating crack + interparticle pore type dominated.

The layer in RMS-2 is dominated by stiff and interprticle pore type that marked by the higher acoustic impedance (40000-60000 (ft.s)/(g/cc)). While 4790-4805 $\mathrm{ft}$ depth shows that the porosity value does not increase significantly and high acoustic impedance as shown in Figure 9.

SAP-1 well is dominated by crack and interparticle pore type and high porosity, yet less clay contains. Structurally, the location of SAP-1 well at an active fault zone, it causes the layer is dominated by crack and interparticle pore type (Fig. 10).

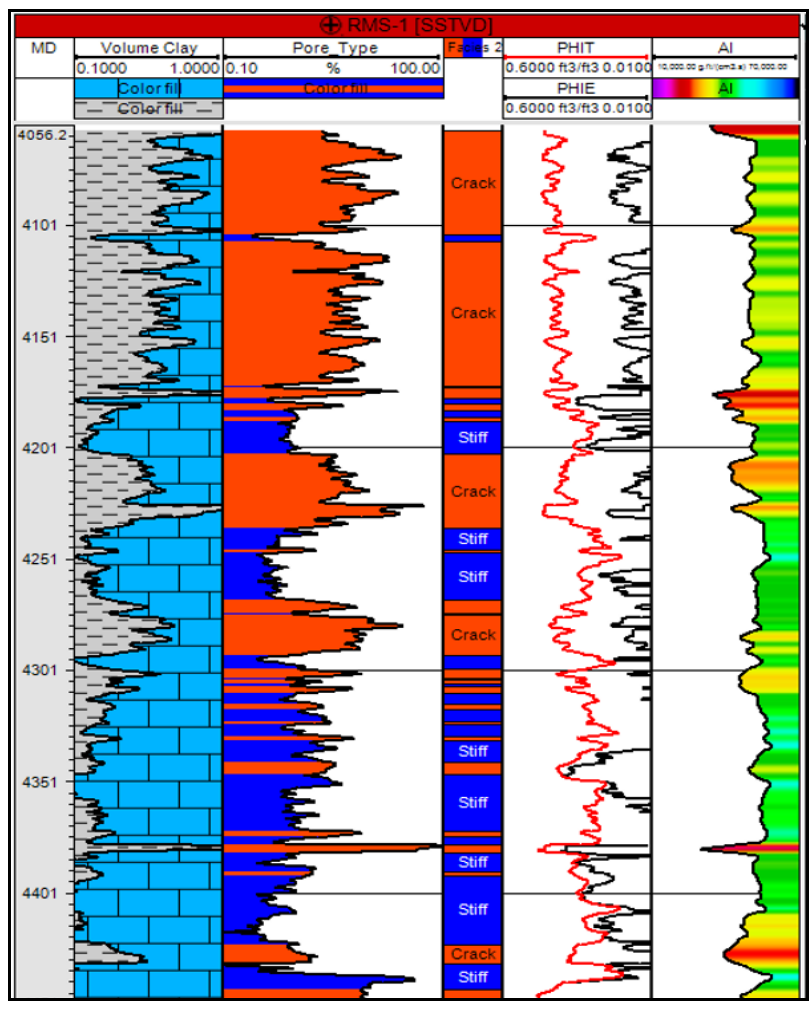

Fig. 8. Volume clay, pore type, porosity, and acoustic impedance log data in well RMS-2 Kais Formation 


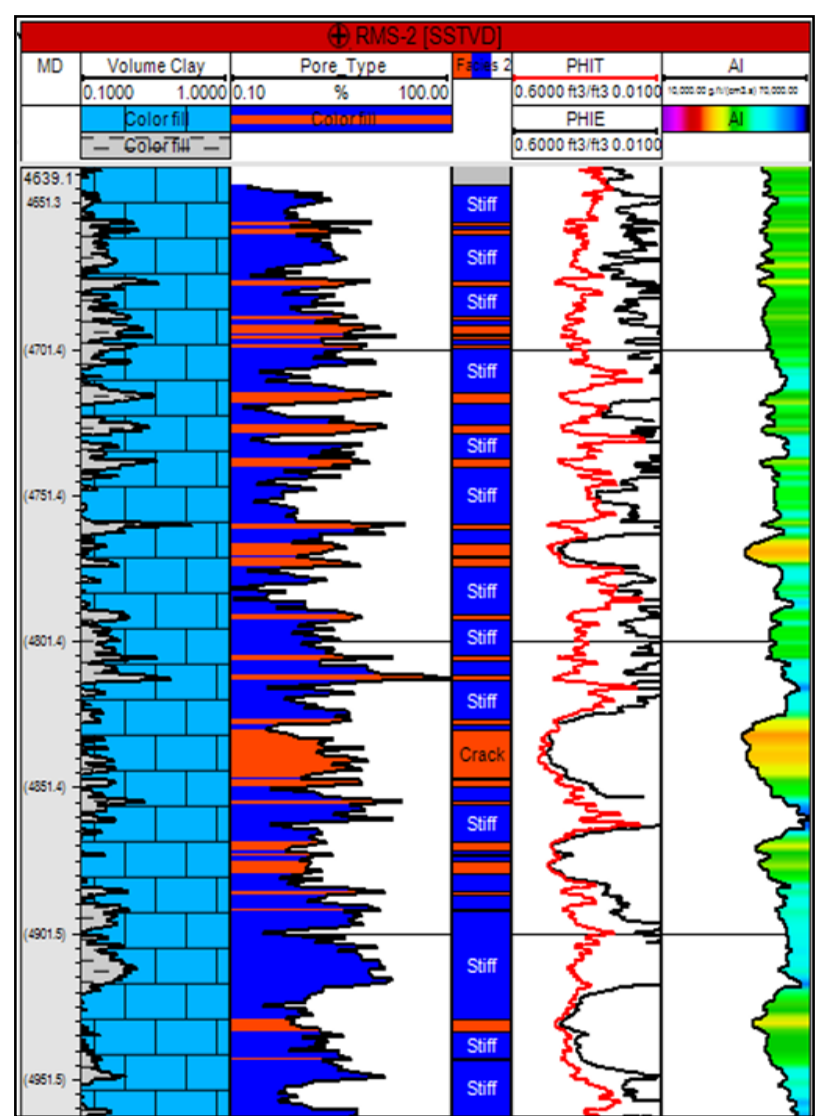

Fig. 9. Volume clay, pore type, porosity, and acoustic impedance log data in well RMS-2 Kais Formation

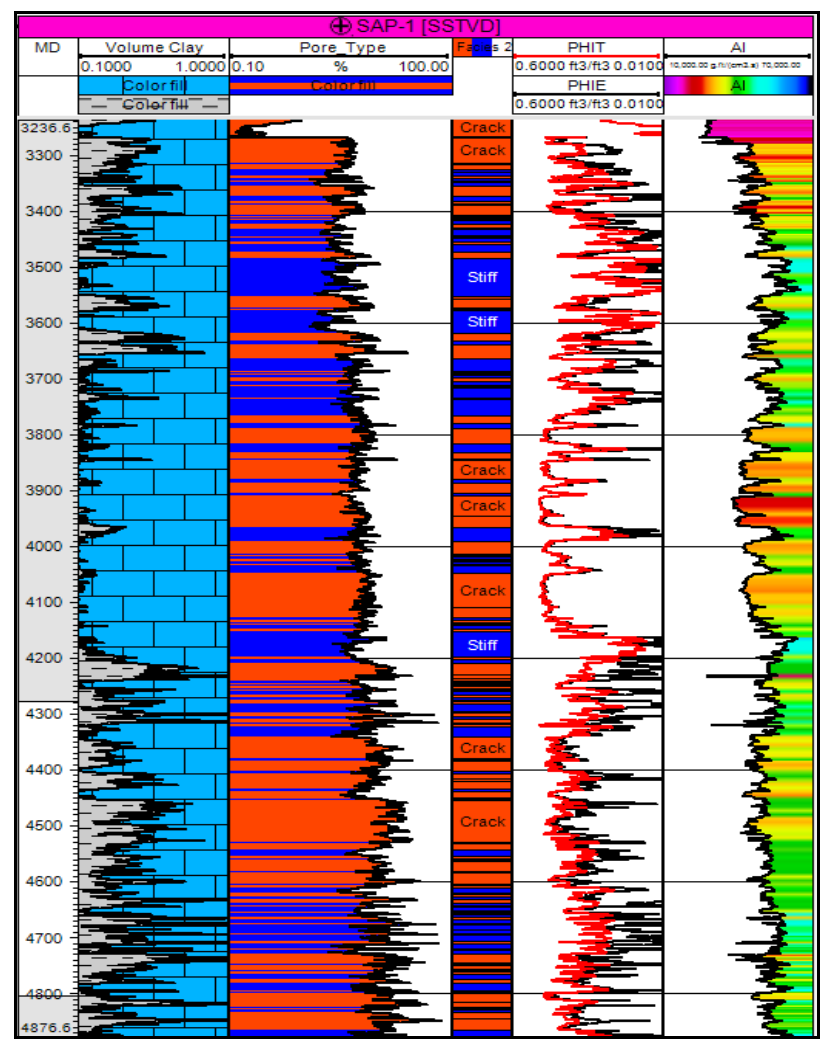

Fig. 10. Volume clay, pore type, porosity, and acoustic impedance log data in well SAP-1 Kais Formation
On the other hand, the Acoustic Impedance value is multiplication between P-wave and density. The degradation of Acoustic Impedance value on crack and interparticle pore type is dominantly affected by $\mathrm{P}$ wave velocity. The $V p$ parameter has rock bulk modulus information, fluid bulk modulus, and rock rigidity. If $\mathrm{P}$ wave velocity exceeds the porous zone, therefore wave velocity will be deviated. The deviation is in the form of velocity degradation. The wave is absorbed by fluid inside pores. Moreover, a relatively high Acoustic Impedance value degradation can be used as a determination parameter to classify carbonate reservoir pore type, vertically or laterally.

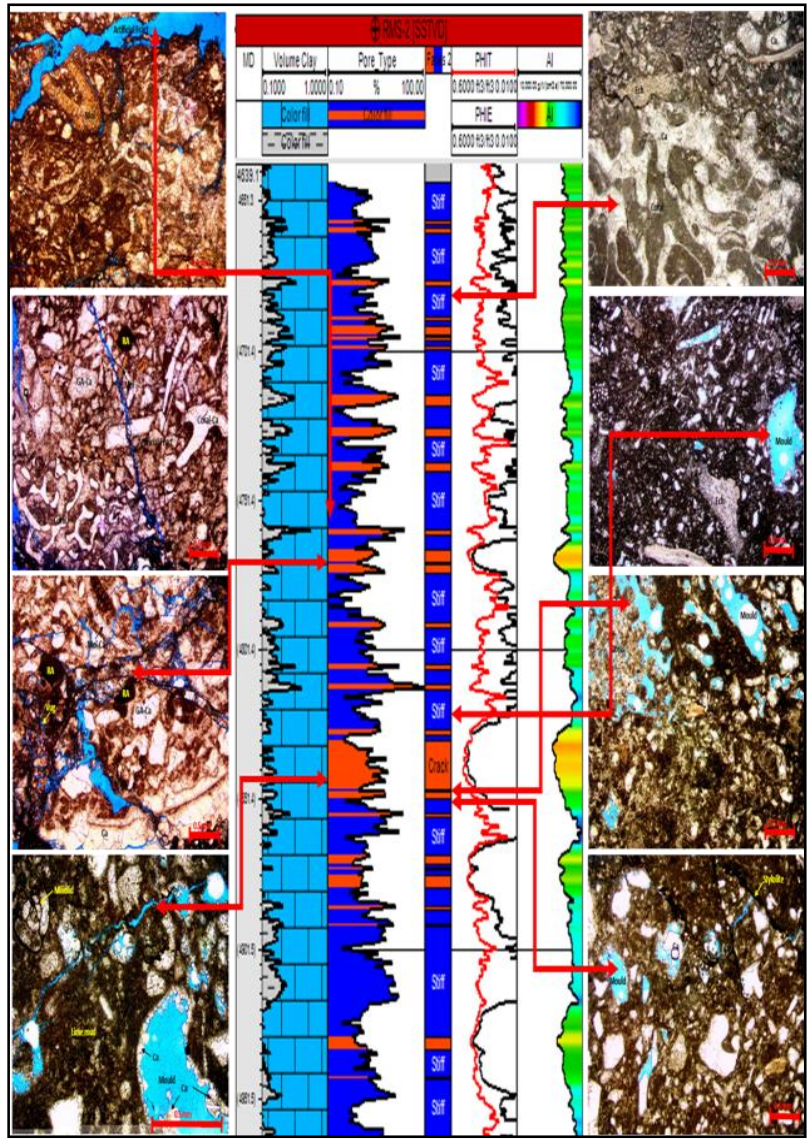

Fig. 11. Thin section of well RMS-2 with crack (open fractured) and stiff (moldic/vuggy) pore type

Commonly, pores inside carbonate can be classified based on matrix composition. Nevertheless, in geophysics standpoint, it is classified as: (1) pore reference, where pores type is assumed as dominant pore inside carbonate. (2) High ratio aspect stiff pore is represented by moldic and vuggy porosity types, which are formed as a result of dissolved grain and skeletal. (3) Lower ratio crack, it is represented by micro crack and micro fracture.

Fig. 11 is RMS-2 petrography data with on the left side is a thin section, which shows open fracture porosity dominated, and on the right side is dominated by vuggy and moldic. Crack and interparticle is correlated with open fracture porosity type, while stiff+interparticle pore type is related to moldic and vuggy porosity types. That data is used to validate DEM method analysis in 
classifying the carbonate reservoir pore type. Based on validation, the DEM method is slightly effective in producing representative pore type with the actual geological condition. This indicates pore moldic/vuggy pores enhanced pore spaces significantly without elastic compressibility of the rock.

\subsection{Pore Type Distribution}

To provide pore type and porosity distribution images, whether laterally or vertically, therefore seismic data is required. Post-stack seismic data needs to be transformed into an elastic parameter by seismic inversion Elastic parameter is in the form of P-impedance (acoustic impedance). As seen in Fig. 8 to Fig. 10, crack pore type shows a degradation of acoustic impedance and porosity significantly. Based on the concept, we identified pore type distribution through the seismic inversion.

Inversion for secondary variable petro physical modeling is model based, it is because the correlation coefficient is higher than other methods. Fig. 12 is an acoustic impedance section through SAP-1, RMS-2, and RMS-1 wells. From the figure, it is known that reef structure impedance value from SAP-1 well is 20000$50000\left((\mathrm{ft} / \mathrm{s})^{*}(\mathrm{~g} / \mathrm{cc})\right)$. While reef structure at RMS-2, the impedance value is $50000-80000((\mathrm{ft} / \mathrm{s}) *(\mathrm{~g} / \mathrm{cc}))$ and reef structure at RMS-1 is $40000-80000\left((\mathrm{ft} / \mathrm{s})^{*}(\mathrm{~g} / \mathrm{cc})\right)$. On Fig.12, it shows increasing of acoustic impedance. Moreover, it is due to the highly reactive chemical from carbonate, and it is constantly cemented, dolomitized, and dissolute. These are affected by temperature, pressure, and water depth. Cementation process also gives effect on acoustic impedance increased, moreover the carbonate is tighter.

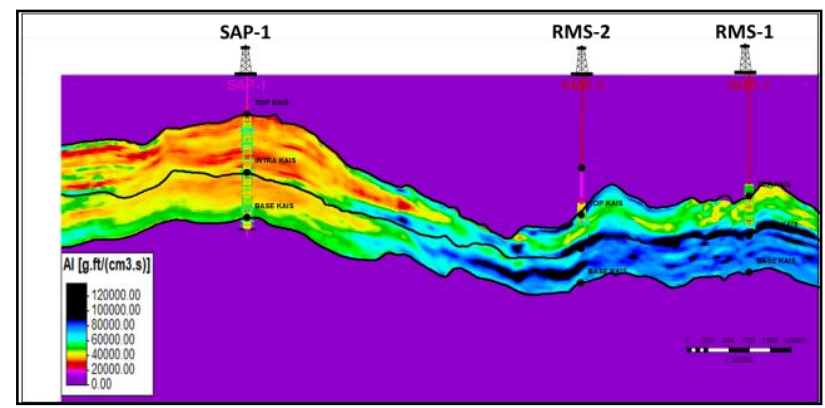

Fig. 12. Section of the acoustic impedance distribution Kais Formation

To obtain pore type distribution and porosity, a geostatistical method such as SGS, stochastic algorithm interpolation, Kriging average, and variance to produce the Gaussian plane. This algorithm is by using data input and data simulation in each grid cell calculation. Input data in the algorithm can be in the form of variogram, 1D vertical direction function, 2D horizontal direction, and 3D property volume. SGS provides several realizations, which can be set based on property value. It is also distributed on a model. The input parameter to distribute pore type and porosity are variogram and 3D AI as trends. Variogram is the most important to show spatial coherence in geostatistical estimation. In variogram determination, it requires a match between data (experimental variogram) with a theoretical variogram (Fig. 13). Variogram that has been used in this study is Gaussian. The input parameter in the variogram determines major and minor directions and true vertical range. On the other hand, the $3 \mathrm{D}$ trend used acoustic impedance, which correlates with pore type and porosity.

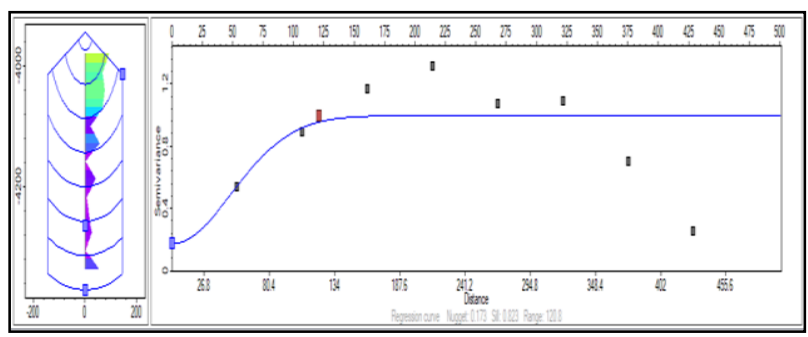

Fig. 13. Variogram Gaussian Model pore type well RMS-1

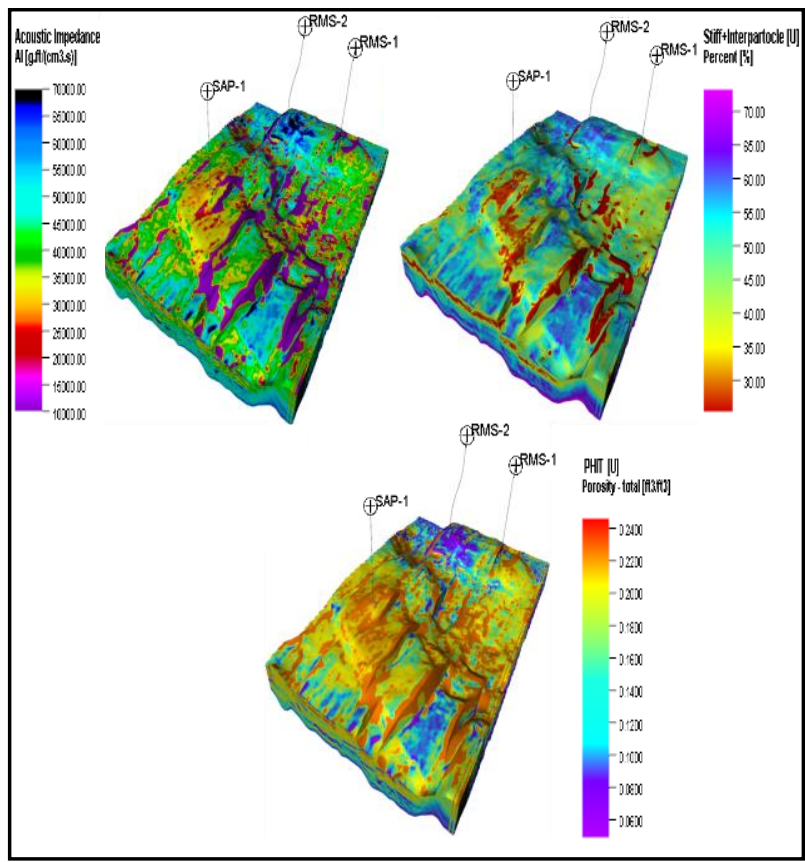

Fig. 14. 3D model acoustic impedance. Pore Type, Porosity

Fig. 14 shows the 3D model of acoustic impedance, stiff and interparticle percentage, and porosity distribution. Overall, the good reservoir from Kais Formation is located in the southern part and dominated by low acoustic impedance, high porosity low stiff and interparticle, and percentage.

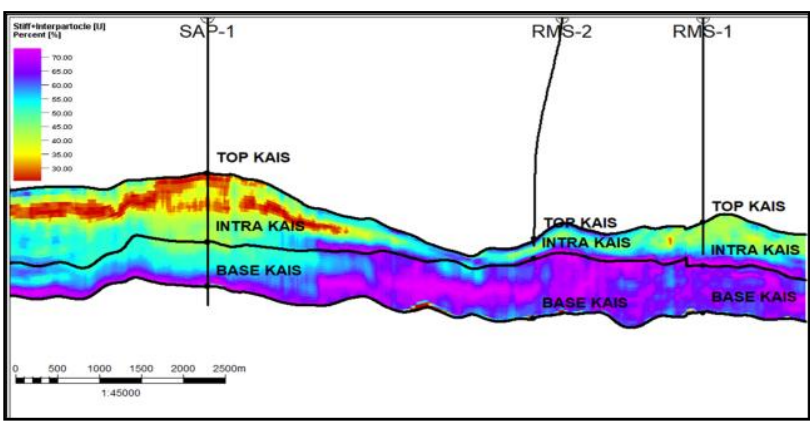

Fig. 15. Section of the Stiff+interparticle precentage distribution Kais Formation 
Fig, 15 shows Stiff and interparticle percentage on reef structure SAP-1 well. It shows $30 \%$ only, therefore pore type has been classified dominantly as crack and interparticle. On the other hand, Fig. 16 shows a high value of porosity at the reef structure SAP-1 and generally, the porosity decreases as depth increasing as well. It is matched that crack and interparticle pore type connects pore spaces, therefore provides a high value of porosity. If we take a closer look, the deeper it gets, it shows decreasing porosity value and dominant Stiff and interparticle pore type percentage. This is due to the diagenesis effect in the form of consolidation, compaction, and cementation. On the other hand, Top Kais Fm has affected the development of fracture on top formation as results of tectonic activity, which formed faults in the "P" field. Therefore, rock on that zone creates a fracture, which enhances porosity value. Tectonic activity developed during Plio-Pleistocene, when Kais. Fm carbonate had been formed and compacted. Fault, which has an instrumental role as displacement zone is Sorong Fault and Sele Fault, therefore local North-South faults, such as at "P" Field (see Fig. 17, Fig. 18, and Fig. 19).

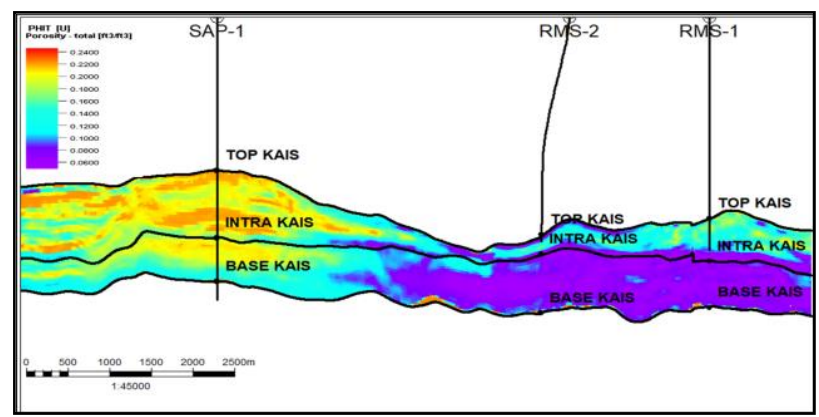

Fig. 16. Section of the porosity fraction distribution Kais Formation

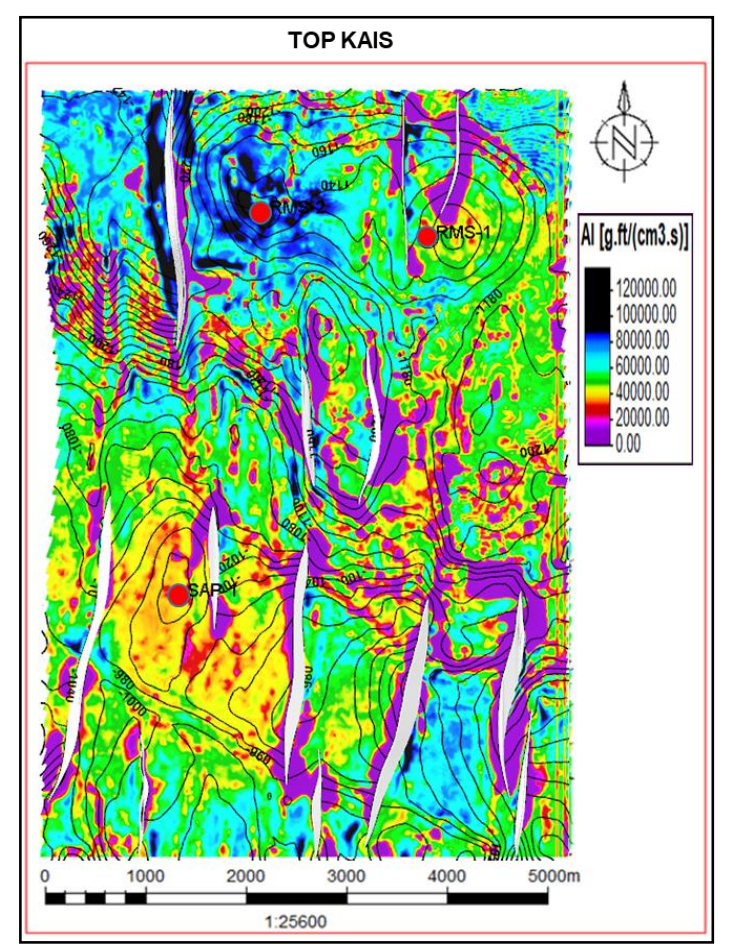

Fig. 17. Map of Acoustic Impedance distribution Top Kais Fm
The top of reef structure SAP-1 and fault zone are dominated low acoustic impedance as well as RMS-1. Whereas, the Acoustic Impedance distribution in reef structure RMS-2 has high value at Top Structure of Kais Formation (Fig. 17).

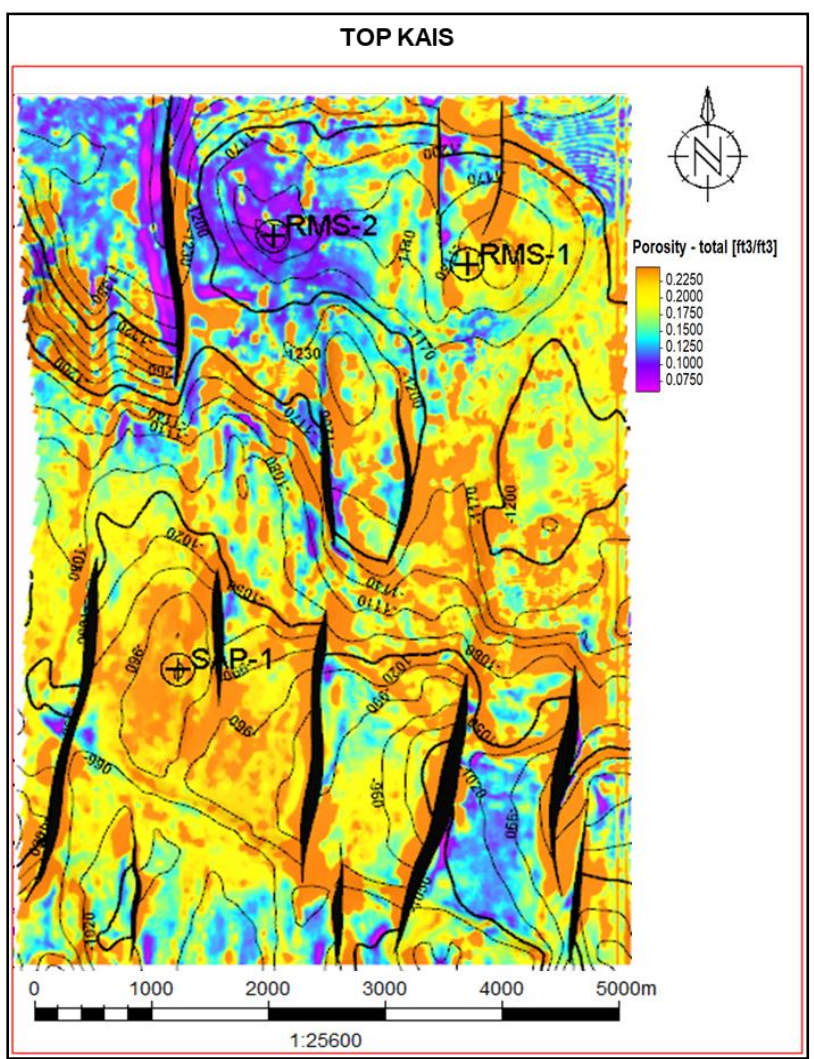

Fig. 18. Map of Porosity distribution Top Kais Fm

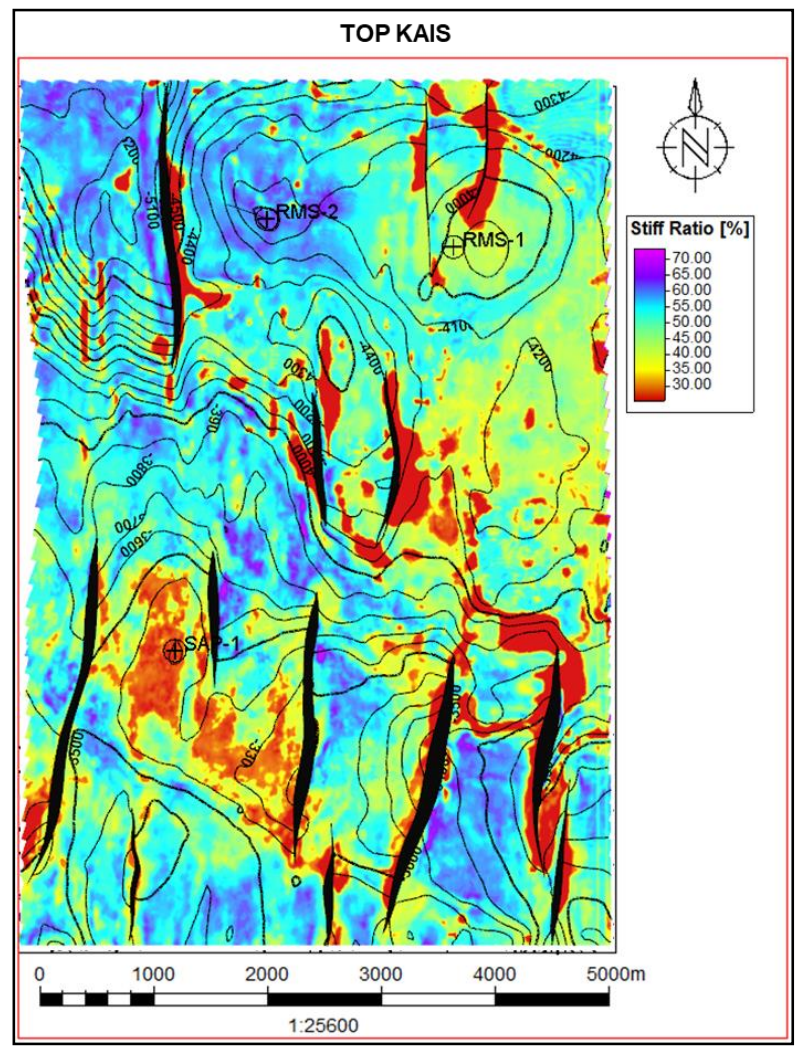

Fig. 19. Map of Stiff and interparticle distribution Top Kais Fm 
Distribution of porosity at fault zone and reef structure SAP-1 is amount $25 \%$. Low porosity distribution is located in reef structure RMS-2 that correlated to the high acoustic impedance at Top Kais Formation. Structurally, porosity distribution in SAP-1, RMS-1, and other reef structure at southern part are a prospect. A low Stiff and interparticle percentage is distributed in top of the reef structure SAP-1 with a value of $30 \%$. So, pore type domination is crack and interparticle. It is also dominating in reef structure RMS1 and fault zone.

Porosity, acoustic impedance, and pore type in carbonate reservoir are influenced by geological aspects. Geologically, reef structure at SAP-1 well is at ramp crest, therefore the lithofacies are packstone and rudstone with high energy. While the northern part of it, which is at reef RMS-2 and RMS-1, it is located at medium ramp with low-medium energy. Therefore the lithofacies are mudstone to wackestone and wackestone to packstone. These are mud-dominated (micrite) or mud supported carbonate rocks and the grains containment is more than $10 \%$. Based on lithology reef carbonate, RMS-2 is containing less matrix than cement of carbonate. It causes low porosity, high acoustic impedance, and Stiff and interparticle pore type dominated in RMS-2.

\section{Conclusion}

The results show there are three pore types indentified, they are stiff pore, interparticle pore, and crack pore. The results of rock physic modelling, RMS-1 and SAP-1 are dominated by crack and interparticle pore type, while RMS-2 is dominated by Stiff and interparticle. The value of acoustic impedance is $25000-35000((\mathrm{ft} / \mathrm{s}) .(\mathrm{g} / \mathrm{cc}))$, it is correlated to value of porosity is $22.5 \%$ and $25 \%-30 \%$ Stiff and interparticle percentage. Pore type and high porosity distribution are influenced by depositional environment process and tectonic activity that marked high porosity and crack and interparticle dominated. Based on the depositional process, crack and interparticle pore type, and high porosity distribution located at crest ramp, part of the southern field "P".

\section{References}

1. A. Hafeez, Developing a Versatile Method for Rock Physics Modeling in a Carbonate Reservoir by Integrating Rock Mechanic Laboratory Results, Petrophysical Analysis and Computational Rock Physics: A Case Study from The Kais Formation in the Salawati Basin (2018)

2. P.G. Pamurty, Identification of Fractured Basement Reservoir in SWO Field, Salawati Basin, West Papua, Based on Seismic Data: A New Challenge and Opportunity for Hydrocarbon Exploration in Pre-Tertiary Basement (2018)

3. L. Zhao, M. Nasser, D. Han, Geophysical Pore Type Characterization from Seismic Data in Carbonate Reservoir, 74th EAGE Conference and
Exhibition incorporating EUROPEC 2012 (2014)

4. F.S. Anselmetti, G.P. Eberli, The VelocityDeviation Log: A Tool to Predict Pore Type and Permeability Trends in Carbonate Drill Holes From Sonic and Porosity or Density Logs, American Assoc. Pet. Geol. Bull. (1999)

5. Y.N. Candikia, M.S. Rosid, M.W. Haidar, Comparative Study Between Kuster-Toksoz and Differential Effective Medium Method for Determining Pore Type in Carbonate Reservoir, AIP Conference Proceedings (2017)

6. M.S. Rosid, S.D. Wahyuni, M.W. Haidar, Carbonate Reservoir Characterization with Pore Type Inversion Using Differential Effective Medium (DEM) Model at ' $x$ ' Field, East Java, AIP Conference Proceedings (2017)

7. C. M. Froidevaux, Tertiary Tectonic History of The Salawati Area, Irian Jaya, Indonesia (2018)

8. A.H. Satyana, Re-Evaluation of the Sedimentology and Evolution of the Kais Carbonate Platform, Salawati Basin, Eastern Indonesia: Exploration Significance (2018)

9. S. Xu, M.A. Payne, Modeling Elastic Properties in Carbonate Rocks, Lead. Edge (2009)

10. J.P. Castagna, M.L. Batzle, K.M. Tubman, J.E. Gaiser, M.D. Burnett, Shear-Wave Velocity Control, Offset-dependent reflectivity - theory Pract. AVO Anal (1993) 